\title{
La influencia del clima de aula sobre las emociones del alumnado The influence of the classroom climate on the student's emotions
}

\author{
Sebastián Fierro-Suero, Natalia Velázquez-Ahumada, Carlos Fernández-Espínola \\ Universidad de Huelva (España)
}

\begin{abstract}
Resumen. En los últimos años, ha aumentado la importancia de las emociones en la educación escolar. La bibliografía actual ha puesto de manifiesto los beneficios de generar climas de aula positivos, en los que existan relaciones afectivas entre los agentes involucrados en el proceso educativo. El presente estudio tuvo por objetivo conocer la relación entre el clima de aula generado y las emociones experimentadas por los estudiantes. La hipótesis planteada fue que el clima de aula generado podría predecir las emociones experimentadas por los estudiantes. Para ello, se utilizó una muestra de 417 alumnos y alumnas de educación secundaria (44.1\% hombres y 55.9\% mujeres) pertenecientes a tres centros educativos de Huelva (Andalucía, España). A estos estudiantes se les preguntó sobre el clima existente en el aula y las emociones experimentadas durante sus clases de educación física. Los resultados del estudio confirman la relación entre el clima de aula y las emociones, destacando el poder predictor de las dimensiones «satisfacción e involucración en el aula» y «relación con el profesor» para predecir las emociones experimentadas por los estudiantes. Dichos resultados permiten poner en valor la importancia de generar climas positivos a través de la creación de vínculos emocionales entre profesores y alumnos. De esta forma, los alumnos y las alumnas se sentirán a gusto y generarán emociones positivas tan importantes en el proceso educativo.
\end{abstract}

Palabras clave: emoción, profesor, estudiante, educación emocional, educación física.

\begin{abstract}
Recently, the importance of emotions in school education has increased. The current literature has highlighted the benefits of generating positive classroom climates, in which there are affective relationships between agents involved in the educational process. This study aimed to know the relationship between the classroom climate generated and the emotions experienced by the students. The hypothesis was that the classroom climate generated could predict the emotions experienced by the students. For this, a sample of 417 secondary school students was used (44.1\% men and 55.9\% women), belonging to three schools located in Huelva (Andalucía, Spain). The students were asked about the existing classroom climate and emotions experienced during their physical education classes. The results of the study confirm the relationship between the classroom climate and emotions. Highlighting the predictive power of the «satisfaction in the classroom» and «relationship with the teacher» dimensions to predict the emotions experienced by students. These results allow us to know the importance of generating positive climates through the creation of emotional bonds between teachers and their students. In this way, students will feel comfortable and will generate positive emotions, which are key factors in the educational process.
\end{abstract}

Key words: emotion, teacher, student, emotional education, physical education.

\section{Introducción}

Las experiencias emocionales están siempre presentes en nuestro día a día, por lo que tienen un papel fundamental en las diferentes situaciones que se dan durante el procesos de aprendizaje (Pekrun, 2006). Hasta hace pocos años, se entendía que la toma de decisiones correcta se basaba en separar emoción y razón. Damasio (2001) mostró que, por un lado, la separación entre emoción y razón es imposible y por el otro, que las emociones jugaban un papel muy importante en la toma de las decisiones. Por este motivo, la investigación sobre emociones en la educación en general (Destacamento, 2018; Pekrun et al., 2011) y en la educación

Fecha recepción: 20-01-21. Fecha de aceptación: 21-04-21

Sebastián Fierro-Suero

fierro.suero@ddi.uhu.es física en particular (Fierro-Suero et al., 2020; Leisterer \& Jekauc, 2019; Mujica, 2021; Mujica \& Jiménez, 2021; Simonton \& Garn, 2019) ha pasado a ser un tema frecuente de estudio en las últimas décadas. Las emociones son constructos complejos y por este motivo es difícil abordar su definición (Vecina, 2006). La literatura científica ha llegado al consenso de que las emociones están compuestas de procesos psicológicos coordinados, que incluyen componentes afectivos, cognitivos, fisiológicos, motivacionales y expresivos (Reeve, 2010). Algunos estudios se han centrado en el papel que juega la inteligencia emocional en la educación física (FernándezEspínola \& Almagro, 2019). Esta se ha asociado a un mayor rendimiento académico, a un mayor bienestar o a menor número de conductas disruptivas entre otros beneficios (Extremera \& Fernández Berrocal, 2003; Fierro-Suero, et al., 2019; Jiménez Morales \& López-Zafra, 2009). Sin embargo, el estudio de las emociones 
concretas que se producen durante el proceso educativo ha sido abordado en menor medida. Aún quedan numerosos tópicos por resolver como las posibles consecuencias de cada una de las emociones, como generar emociones positivas o evitar ciertas emociones negativas en situaciones específicas o como afecta el ambiente generado por los docentes en las emociones de los alumnos.

Una de las teorías principales que está abordando el estudio de las emociones concretas en la educación en general y en la educación física en particular es la Teoría del Control-Valor de las Emociones de Logro («Control-Value Theory of Achievement Emotions-CVTAE») (Pekrun, 2006). Dicha teoría vincula las emociones directamente a la evaluación consciente o inconsciente que se haga de las situaciones específicas que se dan durante la enseñanza (Pekrun \& Stephens, 2010). De este modo, clasifica las emociones en base a tres criterios principales: su valencia (positiva o negativa), su nivel de activación (activadoras o desactivadoras) y el foco del objetivo si es una actividad (e.g. estudiar, tomar apuntes, asistir a una clase, etc) o un resultado (e.g. aprobar o suspender un examen, conseguir reconocimiento por parte del profesor o de sus compañeros, etc) (FierroSuero et al., 2020).

Las emociones positivas que son experimentadas por el alumnado se asocian a una mayor flexibilidad cognitiva y atención, a una mayor salud física y a un mejor rendimiento académico. Por el contrario, las emociones negativas se vinculan con menor atención, reducción del interés y la motivación y con un aprendizaje más superficial (Pekrun et al., 2017; Pekrun et al., 2011; Reschly et al., 2008). Por tanto, uno de los objetivos fundamentales y primordiales de los docentes debería ser generar ambientes y estrategias que promuevan las emociones positivas (Pekrun, 2006). Por ejemplo, en un estudio reciente Fierro-Suero et al. (2020) han mostrado que la creación de climas en los que se satisfagan las necesidades psicológicas básicas podría ayudar a generar emociones positivas y, por otro lado, disminuir las emociones negativas. Además, la expresión, la identificación y la comprensión de las emociones es fundamental para generar interacciones afectivas entre los estudiantes y los profesores (Meyer \& Turner, 2006). Así, en función de las normas que se establezcan, la forma en la que se entienda la disciplina o en la que se organiza la clase, los docentes abordarán de una u otra forma el proceso educativo (Martínez, 2000) generando de esta forma un clima de aula que será de vital importancia.

El concepto de clima de aula es un concepto subjeti- vo e individual que se enfoca desde diferentes perspectivas (Pérez et al., 2009). López y Bisquerra (2013, p.65) lo definieron como «la percepción general, subjetiva y reflexionada que tiene un alumno acerca de las interacciones que se producen en su grupo-clase entre iguales y entre profesor-alumno (...)». Así pues, podría entenderse que este concepto está compuesto de tres variables como son el vínculo docente-alumno, el vínculo entre alumnos y el clima que emerge de esta doble relación entre alumnos y profesores (Casassus, 2008). Todos los adolescentes están obligados a pasar una gran parte del tiempo en la escuela en compañía de sus profesores y compañeros, por lo que ambos acaban influyendo en el correcto desarrollo de los estudiantes (Eccles \& Roeser, 2003). Un clima en el aula positivo se caracteriza por relaciones cálidas, respetuosas y de apoyo emocional. Por el contrario, en aquellas aulas en las que los maestros y los alumnos y alumnas comparten pocas conexiones emocionales, se ignoran, se faltan el respeto, se burlan, etc., se caracterizan por un clima de aula negativo (Reyes et al., 2012). Esta relación entre los estudiantes y los profesores, ha sido la más estudiada debido a su gran importancia (e.g. Denham et al., 2012; Jiang et al., 2016; Mejía \& Arturo, 2009; Moreno-Murcia et al., 2018). El docente debe ser emocionalmente competente, de tal forma que sea capaz de entender las individualidades personales para que todos los alumnos se sientan comprendidos y respetados (Casassus, 2008). Además, los alumnos son capaces de detectar las emociones que están siendo experimentadas por sus profesores pudiendo llegar a producirse contagios emocionales entre el profesorado y el alumnado (Frenzel et al., 2009; Jiang et al., 2016). Por este motivo, ha aumentado el número de investigaciones centradas en conocer las emociones que experimentan los profesores (e.g. Fierro-Suero et al., 2021; Luo et al., 2019) ya que estas juegan un papel fundamental en la calidad de la enseñanza de los docentes (Becker et al., 2014; Chen, 2019), así como en el aprendizaje y en la motivación de los alumnos (Arens \& Morin, 2016; Løvoll et al., 2017, 2020).

El apoyo social es un predictor del bienestar psicológico en los niños y en los jóvenes (Musitu \& Cava, 2003). Concretamente, los alumnos y alumnas a los que se les da suficiente apoyo emocional por parte de sus profesores parecen expresar mayor interés, más disfrute, mayor compromiso y mejor desempeño académico (Brock et al., 2008; Reyes et al., 2012). Para que esto ocurra es fundamental que se preste especial atención a los aspectos sociales y emocionales del aprendizaje, ade- 
más de fomentar el disfrute, expresar interés por el alumnado e incentivar la cooperación entre ellos (Reyes et al., 2012). La creación de relaciones sociales entre iguales ha mostrado ser una variable importante para generar un clima positivo en el aula (HombradosMendieta \& Castro-Travé, 2013). Para conseguir el apoyo social entre iguales se requiere de una participación extensa y continua (Casassus, 2008). Los alumnos y alumnas que se sienten socialmente aceptados presentan un mayor rendimiento académico y una mayor autoestima, entre otros muchos beneficios (Berger et al., 2014).

La bibliografía planteada anteriormente muestra algunas evidencias de la posible relación entre el clima de aula y las emociones experimentadas por los agentes involucrados en el proceso educativo (profesores y alumnos) como se ha puesto de manifiesto previamente con los climas motivacionales (Baños \& Arrayales, 2020). Por este motivo, la finalidad del presente trabajo es conocer de forma objetiva y con mayor exactitud la relación entre el clima de aula y las emociones experimentadas por los alumnos y las alumnas durante las clases de educación física. Además, se quiere comprobar si el clima de aula puede actuar como predictor de estas emociones. Para ello, la hipótesis principal es que el clima de clase podría predecir las emociones de los estudiantes.

\section{Método}

\section{Participantes}

La muestra del estudio estuvo compuesta por 474 alumnos y alumnas pertenecientes a tres Institutos de Educación Secundaria Obligatoria de la provincia de Huelva (Andalucía, España). Los participantes fueron 209 hombres (44.1\%) y 265 mujeres (55.9\%) con edades comprendidas entre los 11 y los 17 años con una media de 13.10 años $(D T=1.22)$.

\section{Instrumentos}

Escala Breve de Clima de Clase (EBCC). La escala creada por López y Bisquerra (2013) está compuesta por diferentes dimensiones que miden el clima de clase. En este caso se usaron las dimensiones satisfacción e involucración (e.g. «estoy a gusto en esta clase), cohesión entre iguales (e.g. «los alumnos nos ayudamos unos a otros») y relación profesor-alumno (e.g. «la relación con el profesor es buena»). Las respuestas fueron puntuadas en una escala tipo Likert con un rango de puntuación que oscila de 1 (nunca) a 4 (siempre). Los alfas de Cronbach obtenidos fueron .71 para la satisfac- ción e involucración, .73 para la cohesión entre iguales y .82 para la relación profesor-alumno.

Achievement Emotions Questionnaire for Physical Education (AEQ-PE). Se utilizó la versión validada al castellano de Fierro-Suero et al. (2020). Este instrumento basado en la CVTAE está compuesto de 24 ítems que miden 6 emociones. Las emociones estudiadas son: disfrute (e.g. «disfruto estando en clase de educación física»), orgullo (e.g. «como me siento orgulloso de mis logros en educación física, estoy motivado para continuar «), aburrimiento (e.g. «tengo ganas de que termine la clase de educación física porque es muy aburrida»), ira (e.g. «siento un enfado que va creciendo en mi interior durante la clase de educación física»), ansiedad (e.g. «me da miedo poder decir/hacer algo incorrecto en clase de educación física, preferiría no decir/hacer nada «) y desesperanza (e.g. «he perdido toda esperanza de hacer eficazmente las actividades de educación física «) . Las respuestas fueron puntuadas en una escala tipo Likert con un rango de puntuación que oscila de 1 (totalmente en desacuerdo) a 5 (totalmente de acuerdo). Los alfas de Cronbach obtenidas fueron .85 para el disfrute, .78 para el orgullo, .81 para el aburrimiento, .71 para la ira, .72 para la ansiedad y .81 para la desesperanza.

\section{Procedimiento}

El estudio realizado cuenta con la aprobación del comité Andaluz de Investigación Biomédica (TDOCME-2018) y fue llevado a cabo siguiendo los principios éticos establecidos por la Asociación Americana de Psicología (American Psychological Association, 2010).

En primer lugar, se procedió a contactar con los equipos directivos de los diferentes centros educativos seleccionados con el fin de darles la información necesaria para la realización del estudio, además de informarles sobre el objetivo final de dicho estudio y finalmente pedir su participación y colaboración en él. Debido a que los alumnos y alumnas participantes tenían edades comprendidas entre los 11 y los 17 años, se solicitó autorización a los responsables legales para su participación en el estudio.

Una vez se contaba con los permisos y autorizaciones necesarias se procedió a la recogida de datos, estos fueron tomados durante los meses de noviembre y diciembre de 2019, siempre en horario escolar y en presencia del tutor del curso. Durante la recogida de datos el investigador estaba presente para poder aclarar las diferentes dudas que surgiesen y responder las preguntas de los participantes. El tiempo aproximado para com- 
pletar las diferentes escalas fue de 10 minutos.

La participación en el estudio fue voluntaria, y se aclaró que se podía abandonar el estudio en el momento que así lo desease el participante.

\section{Análisis de datos}

En primer lugar, se procedió a volcar los datos en una base de datos conjunta para, posteriormente, poder estudiar los estadísticos descriptivos, así como la fiabilidad de las diferentes escalas a través del alfa de Cronbach. A continuación, se examinaron las relaciones entre las variables del estudio (cada una de las emociones y cada una de las dimensiones del clima de aula) mediante correlaciones bivariadas. Por último, se propusieron dos análisis de regresión lineal múltiple en un único paso para conocer si las diferentes dimensiones del clima de aula podían actuar como variables predictoras de las emociones expresadas por los alumnos y las alumnas. Para el análisis de los datos se utilizó el programa estadístico SPSS en la versión 23.0 (IBM, Armonk, NY, USA).

\section{Resultados}

Tanto las diferentes emociones estudiadas como las dimensiones del clima de aula mostraron una adecuada consistencia interna con valores en el alfa de Cronbach por encima de los recomendados (.70) (Tabla 1). A nivel descriptivo (Tabla 1) las emociones positivas (disfrute y orgullo) fueron las emociones que presentaron los valores más altos, estando en ambos casos cercanos 4 en una escala de 1 a 5 . Por su parte, las emociones negativas que expresaron sentir en mayor medida los alumnos durante sus clases de educación física fueron la ansiedad, el aburrimiento, la ira y la desesperanza por este orden. Además, las diferentes dimensiones del clima de aula (satisfacción e involucración en clase, cohesión entre iguales y relación con el profesor) han mostrado valores similares entre ellas.

El análisis de las correlaciones bilaterales (Tabla 1) mostró que las emociones positivas correlacionaban de forma positiva entre ellas y con las dimensiones del clima de aula estudiadas. Por el contrario, las emociones positivas correlacionaban de forma negativa con las emociones negativas. Por otro lado, las emociones negativas mostraron correlaciones únicamente de forma positiva entre ellas, y de forma negativa con las emociones positivas y el clima de clase. Por último, las diferentes dimensiones del clima de clase correlacionaron de forma positiva entre ellas y con las emociones de valencia positivas, y de forma negativa con las emociones de valencia negativas. Todas las correlaciones fueron estadísticamente significativas a excepción de la relación entre la ira y con la dimensión «relación con el profesor».

Tabla 1

Estadísticos descriptivos y correlaciones de las variables de estudi

\begin{tabular}{|c|c|c|c|c|c|c|c|c|c|c|}
\hline & M & DT & 122 & 3 & 4 & 5 & 6 & 7 & 8 & 9 \\
\hline Disfrute & 3.93 & .98 & $-.73 * *$ & $-.55 * *$ & $-.44 * *$ & $-.18 * *$ & $-.46 * *$ & $.40 * *$ & $.31 * *$ & $.45 * *$ \\
\hline Orgullo & 3.97 & .88 & - & $-.72 * *$ & $-.55 * *$ & $-.14 * *$ & $-.51 * *$ & $.46 * *$ & $.32 * *$ & $.50 * *$ \\
\hline Aburrimiento & 1.95 & .93 & & & $.47 * *$ & $.10 *$ & $.55 * *$ & $-.38 * *$ & $-.25 * *$ & $-.46 * *$ \\
\hline 4. Desesperanza & 1.49 & .70 & & & & $.41 * *$ & $.58 * *$ & $-.33 * *$ & $-.25 * *$ & $-.34 * *$ \\
\hline Ira & 1.51 & .69 & & & & - & $.33 * *$ & $-.22 * *$ & $-.17 * *$ & -.05 \\
\hline Ansiedad & 2.00 & .87 & & & & & - & $-.39 * *$ & $-.30 * *$ & $-.33 * *$ \\
\hline Clima satisfacción en clase & 3.20 & .72 & & & & & & - & $.58 * *$ & $.52 * *$ \\
\hline Clima cohesión entre iguales & 3.28 & .69 & & & & & & & & $.46 * *$ \\
\hline Clima relación profesor & 3.29 & .79 & & & & & & & & - \\
\hline
\end{tabular}

$\begin{array}{lll}\text { 9. Clima relación profesor } \quad 3.29 & .79\end{array}$

Nota. a $=$ Alfa de Cronbach; $\mathrm{M}=$ Media; DT $=$ Desviación Típica; $* \mathrm{p}<.05 * * \mathrm{p}<.01$

Con la finalidad de simplificar los resultados del análisis de regresión, en base a los objetivos del estudio y los resultados obtenidos de los análisis descriptivos, se decidió unificar los tipos de emociones según su valencia (positivas y negativas). Por lo que se propusieron dos análisis de regresión separados (Tabla 2) para conocer en qué medida las dimensiones del clima de aula podían predecir las emociones generadas (positivas o negativas). Finalmente, tanto las emociones positivas (32\% de la varianza explicada) como las emociones negativas ( $24 \%$ de la varianza explicada) fueron predichas por el clima de aula. Concretamente, la dimensión «relación con el profesor» fue la que obtuvo un mayor peso de regresión para las emociones positivas y la dimensión «satisfacción e involucración en el aula» para las emociones negativas. Por último, hay que mencionar que la dimensión cohesión entre iguales no predijo las emociones experimentadas por los estudiantes (ni positivas, ni negativas).

Tabla 2.
Análisis de regresión lineal

\section{Discusión}

Existen diferentes estudios que han mostrado la importancia de las emociones en el proceso educativo (Pekrun et al., 2017; Pekrun, 2006) jugando en este proceso un papel fundamental el clima de aula generado (Brock et al., 2008; Martínez, 2000). Dentro de los climas de aula, las relaciones socioafectivas tienen un rol muy importante (Casassus, 2008; Musitu \& Cava, 2003) que deberán partir de la expresión, la identificación y la 
comprensión de las emociones (Meyer \& Turner, 2006).

En el presente estudio se ha tenido como objetivo principal estudiar en mayor profundidad y con objetividad la relación entre el clima de aula y las emociones experimentadas, examinando las correlaciones existentes entre las dimensiones del clima de aula (satisfacción e involucración en la clase, relación con el profesor y relación entre iguales) y cada una de las emociones estudiadas por el AEQ-EF (Fierro-Suero et al., 2020) basado en la teoría del control-valor de las emociones de logro (CVTAE) (Pekrun, 2006).

Los resultados de estas relaciones indican que todas las emociones con la misma valencia (positiva o negativa) actúan de forma similar, lo que ocurre igualmente con las tres dimensiones estudiadas del clima de aula (satisfacción e involucración en clase, cohesión entre iguales y relación con el profesor). Este hecho sirvió para simplificar los análisis que se realizaron posteriormente. Las relaciones que se halladas son positivas entre las emociones positivas y las dimensiones del clima de clase. Por el contrario, las emociones negativas se correlacionan de forma negativa con el clima en el aula. Estas relaciones son coherentes, y están en la línea de lo esperado en base los a resultados anteriores que señalaron la importancia de las interacciones afectivas entre los individuos involucrados en el proceso educativo (Casassus, 2008; Meyer \& Turner, 2006). Las variables que se integran dentro de un mismo constructo (emociones positivas, emociones negativas y climas de aula) presentan relaciones muy similares, lo que también ha ocurrido en estudios previos (Fierro-Suero et al., 2020; López \& Bisquerra, 2013). A la hora de entrelazar los diferentes constructos (clima de aula y emociones) se esperaba que las relaciones encontradas fueran más específicas, pudiendo diferenciar entre emociones concretas y dimensiones del clima de aula. Esto si ha ocurrido, por ejemplo, en el caso de la emoción «ira» y su relación con la dimensión del clima de clase «relación con el profesor». Dicha relación ha sido la única que no ha presentado correlación estadísticamente significativa. Por este motivo, en futuras investigaciones se plantea que sería conveniente ampliar el abanico de emociones estudiadas, así como el número de dimensiones del clima de aula. Este hecho, podría ayudar y dar a conocer relaciones desconocidas hasta ahora.

Una vez conocida la relación entre las variables del estudio y abordando el segundo objetivo, se procedió a contrastar la hipótesis planteada: el clima de clase predecirá las emociones de los alumnos y las alumnas. Para ello, teniendo en cuenta los resultados obtenidos pre- viamente, se propusieron dos análisis de regresión lineal simplificando los constructos emocionales en función de la valencia emocional, es decir, separando en emociones positivas (disfrute y orgullo) y emociones negativas (aburrimiento, desesperanza, ira y ansiedad). En ambos casos, el clima de clase ha mostrado predecir las emociones experimentadas por los alumnos y las alumnas (con una mayor varianza explicada para las emociones positivas que para las negativas). Por el contrario, las tres dimensiones del clima de clase estudiadas (satisfacción e involucración en la clase, cohesión entre iguales y relación con el profesor) no presentan comportamientos similares. Las dimensiones satisfacción e involucración en el aula y relación con el profesor si predice las emociones experimentadas por los estudiantes, sin embargo, la dimensión cohesión entre iguales no predice dichas emociones. Por tanto, aunque las relaciones sociales entre iguales sea una variable de gran importancia para generar climas positivos (Hombrados-Mendieta \& Castro-Travé, 2013), esta parece que no afecta hacia las emociones concretas que los alumnos expresan sentir durante sus clases de educación física. Esto se puede deber a que emocionalmente a los alumnos les afecta más tener el respaldo de sus profesores que el respaldo de sus compañeros. La relación docente-discente ha sido una de las más estudiadas desde diferentes perspectivas destacando en la bibliografía como la más importante para establecer un clima de aula positivo (Denham et al., 2012; Jiang et al., 2016; Mejía \& Arturo, 2009; Moreno-Murcia et al., 2018; Mujica \& Orellana, 2021). Por este motivo, se debe continuar investigando, en esta línea, sobre las estrategias concretas que ayuden a los docentes a establecer climas positivos de aula y generar emociones positivas en el alumnado. Recientemente Fierro-Suero et al. (2020) han mostrado como la satisfacción de las necesidades psicológicas básicas (autonomía, competencia, relación y se tuvo también en cuenta la novedad como posible cuarta necesidad), actuaban como predictoras de las emociones expresadas por los alumnos y alumnas. Este hecho ha sido igualmente hallado en docentes de diferentes etapas y materias (Fierro-Suero et al., 2021). Por tanto, la satisfacción de estas necesidades puede ser una estrategia que afecte al clima y debería ser tenida en cuenta a la hora de diseñar las sesiones educativas desde el punto de vista emocional, además del motivacional ya mostrado por diversos estudios (Amado et al., 2014; Cheon et al., 2012). Así pues, se plantea necesario, que al igual que ocurre en otros constructos psicológicos, como el caso de la motivación 
con la Teoría de la Autodeterminación (Deci \& Ryan, 1985; Ryan \& Deci, 2017) se investigue sobre las estrategias específicas para desarrollar emociones positivas. Además, también es fundamental que se profundice en conocer cómo afecta el contexto a las emociones experimentadas por el alumnado y como no, conocer con mayor detalle, además de las emociones experimentadas, las consecuencias de cada una de las estrategias desarrolladas para generar emociones. Para ello, es fundamental el desarrollo y aplicación de intervenciones bien fundamentadas y organizadas que arrojen datos concretos y extrapolables a otros contextos, como pueden ser otras asignaturas o las actividades extraescolares.

En el presente trabajo se ha contado con una serie de limitaciones. A pesar de que el abanico de emociones estudiadas es amplio y con respaldo teórico (FierroSuero et al., 2020; Pekrun, 2006), no se han encontrado diferencias destacables entre las emociones con la misma valencia (positivas o negativas). Por esta razón, en futuras ocasiones podría extenderse el número de emociones estudiadas y el número de dimensiones del clima de aula, y de esta forma poder seguir clarificando la importancia del clima sobre las emociones de los alumnos y las alumnas. Además, el estudio se ha centrado en un contexto concreto como es el clima generado durante las clases de educación física. Dada la naturaleza de esta materia, caracterizada por el impacto emocional en los estudiantes (Simonton et al., 2017), puede que las relaciones que se encontrasen en otras materias sean diferentes, por lo que sería una nueva variable a estudiar en ocasiones futuras. De igual forma, se recomienda que, en próximos trabajos, se trate de estudiar si el género puede ser una variable a tener en cuenta y que chicos y chicas experimenten diferentes emociones y climas de aula. Tradicionalmente se cree que las chicas se caracterizan por ser más emocionales, aunque posiblemente se trate de estereotipos o prejuicios establecidos al género femenino (Baron-Cohen, 2005). Igualmente, los cambios experimentados a consecuencia de la edad podrían ser estudiados en futuras investigaciones.

Además, los estudios en esta línea podrían contribuir a crear programas e intervenciones eficaces e individualizados para prevenir problemas emocionales durante la etapa escolar. Las diferentes investigaciones desarrolladas por Achenbach en la década de los 80 mostraron como, los problemas emocionales como pueden ser miedo, apatía o tristeza (síntomas internalizantes) a pesar de su infraestimanción en el diagnóstico, eran más comunes que los síntomas externalizantes (conductas disruptivas, delictivas, etc.) (Achenbach, 1982; Achenbach \& Edel-brock, 1983; Siu, 2008). Además, esta falta de abordaje durante la niñez hace que puedan extenderse y agravarse durante la edad adulta (Achenbach, et al., 2002; Angold, et al., 1998; Costello \& Angold, 1995). Por este motivo resulta importante abordarlos en la edad más temprana posible, al fin y al cabo, la salud emocional de los alumnos y las alumnas debería ser un objetivo educativo por sí mismo (Pekrun, 2014). La educación física se caracteriza por la capacidad para impactar emocionalmente al alumnado (Simonton et al., 2017), lo que debe ser aprovechado para abordar este tipo de problemas. Esta materia está cambiando su enfoque desde lo fisiológico hacia un enfoque mucho más global, donde lo mental tiene cada vez mayor peso (Bermudez \& Saenz-López, 2019). En esta línea, el clima de aula generado debe adquirir un papel fundamental ya que será clave en las emociones experimentadas por los estudiantes. Este un tema que necesita de más estudios, por lo que sería interesante continuar futuras investigaciones en esta dirección complementaria.

\section{Conclusión}

En la presente investigación se ha comprobado la existencia de relación entre las dimensiones del clima de aula estudiadas (satisfacción e involucración en la clase, relación con el profesor y relación entre iguales) y las emociones percibidas por los alumnos y las alumnas. La única relación concreta no estadísticamente significativa se halla entre la ira y la relación con el profesor. Por otro lado, se ha demostrado el poder predictor del clima de aula sobre las emociones (positivas o negativas) tal y como se había hipotetizado. A pesar de que la dimensión cohesión entre iguales del clima de clase correlaciona con todas las emociones, no tiene suficiente peso de regresión para predecirlas. Por este motivo, las dimensiones satisfacción e involucración el aula y relación con el profesor parecen ser más importantes para generar emociones en los alumnos y las alumnas. En base a los resultados obtenidos se plantea fundamental establecer climas positivos de aula en base a estrategias que acerquen la figura de profesores y alumnos ayudándolos a establecer vínculos emocionales que hagan que los alumnos se sientan más satisfechos e involucrados en las clases. Esto hecho, ayudará a la generación de emociones positivas y evitar emociones negativas en los estudiantes, lo que podría tener un gran número de consecuencias positivas académicas y personales. 


\section{Apoyos}

La realización de este trabajo fue posible gracias a las ayudas del programa de Formación del Profesorado Universitario (FPU18/04855) del Ministerio de Ciencias, Innovación y Universidades. Gobierno de España.

El estudio pertenece al proyecto titulado: En busca del bienestar y rendimiento académico a través de la motivación y las emociones en el aula. Entidad Financiadora: Programa Operativo FEDER Andalucía 2014-2020 (proyectos I+D+i). Código: UHU-1259909. Referencia PID2019-106918GB-I00.

\section{Referencias}

Achenbach, T. M. (1982). Developmental psychopathology. Nueva York: John Wiley \& Sons.

Achenbach, T. M., \& Edelbrock, C. S. (1983). Manual of Child Behavior Check-list and Revised Child Behavior Profile. Burlington: University of Vermont, Department of Psychiatry.

Achenbach, T. M., \& Dumenci, L (2001). Advances in Empirically Based Assessment: Revised CrossInformant Syndromes and New DSM - Oriented Scales for the CBCL, YSR, and TRF: Comment on Lengua, Sadowksi, Friedrich, \& Fisher. Journal of Consulting and Clinical Psychology, 69 (4), 699-702.

Amado, D., Del Villar, F., Leo, F. M., Sánchez-Oliva, D., Sánchez-Miguel, P.A., \& García-Calvo,T. (2014). Effect of a multi-dimensional intervention programme on the motivation of physical education students. PLoS ONE, 9(1), 1-6. https://doi.org/ 10.1371 /journal.pone.0085275

American Psychological Association. (2010). Publication Manual of American Psychological Association (6th ed). Washington, DC, USA.

Angold, A., Messer, S.C., Stangl, D., Farmer, E. Costello, E.J. \& Burns, B.J. (1998). Perceived parental burden and service use for child and adolescent psychiatric disorders. American Journal of Public Health, 88, 75-80.

Arens, A. K., \& Morin,A. J. S. (2016). Relations Between Teachers' Emotional Exhaustion and Students' Educational Outcomes. Journal of Educational Psychology, 108(6), 800-813. https://doi.org/ 10.1037/edu0000105.supp

Baños, R., \& Arrayales, E. (2020). Predicción del aburrimiento en la educación física a partir del clima motivacional (Prediction of boredom in physical education from the motivational climate). Retos.
Nuevas tendencias en Educación Física, Deporte y Recreación, 2041(38), 83-88. https://doi.org/10.47197/ retos.v38i38.74301

Baron-Cohen, S. (2005). The essential difference: the male and female brain. Phi Kappa Phi Forum, 85, 2226.

Becker, E. S., Goetz, T., Morger, V., \& Ranellucci, J. (2014). The importance of teachers' emotions and instructional behavior for their students' emotions An experience sampling analysis. Teaching and Teacher Education, 43, 15-26. https://doi.org/10.1016/ j.tate.2014.05.002

Berger, C., Álamos, P., Milicic, N., \& Alcalay, L. (2014). Rendimiento académico y las dimensiones personal y contextual del aprendizaje socioemocional: Evidencias de su asociación en estudiantes chilenos. Universitas Psychologica, 13(2), 627-638. https:// doi.org/10.11144/Javeriana.UPSY13-2.radp

Bermudez, C., \& Saenz-López, P. (2019). Emotions in physical education. A bibliographic review (20152017). Retos. Nuevas tendencias en Educación Física, Deporte y Recreación, 36(2), 597-603.

Brock, L. L., Nishida, T. K., Chiong, C., Grimm, K. J., \& Rimm-kaufman, S. E. (2008). Children's perceptions of the classroom environment and social and academic performance/ : A longitudinal analysis of the contribution of the Responsive Classroom approach. Journal of School Psychology, 46, 129-149. j.jsp.2007.02.004

Casassus, J. (2008). Aprendizajes, emociones y clima de aula. Paulo Freire. Revista de Pedagogía Crítica, 7(6), 8195.

Chen, J. (2019). Exploring the impact of teacher emotions on their approaches to teaching: A structural equation modelling approach. British Journal of Educational Psychology, 89(1), 57-74. https://doi.org/ 10.1111/bjep. 12220

Cheon, S. H., Reeve, J., \& Moon, I. S. (2012). Experimentally based, longitudinally designed, teacher-focused Intervention to help physical education teachers be more autonomy supportive toward their students. Journal of sport \& exercise psychology Exercise Psychology, 34, 365-396. https:// doi.org/10.1123/jsep.34.3.365

Costello, E.J. \& Angold, A. (1995). Developmental Epidemiology. En D. Cichetti \& D.J. Cohen (Eds.), Developmental Psychopathology:Theory and Methods. New York: Wiley Interscience.

Damasio, A. R. (2001). El error de Descartes. Barcelona: 
Crítica.

Deci, E. L., \& Ryan, R. M. (1985). Intrinsic Motivation and Self-Determination in Human Behavior. New York, USA: Plenum.

Denham, S. A., Bassett, H. H., \& Zinsser, K. (2012). Early childhood teachers as socializers of young children's emotional competence. Early Childhood Education Journal, 40(3), 137-143. https: / / doi.org/ $10.1007 / \mathrm{s} 10643-012-0504-2$

Destacamento, R. (2018). Academic Emotions And Performance of the Senior High School Students: Basis for Intervention Program. SMCC Higher Education Research Journal, 5, 69-92. https: / / doi.org/10.18868/ sher5j.05.01213.04

Eccles, J. S., \& Roeser, R. W. (2003). Schools as developmental contexts. En G. Adams \& M.D. Berzonsky (Ed.), Blackwell Handbook of Adolescence (pp. 129-148). Malden, MA: Blackwell Publishing.

Extremera, N., \& Fernández Berrocal, P. (2003). La inteligencia emocional en el contexto educativo/ : hallazgos científicos de sus efectos en el aula. Revista de educación, 332, 97-116.

Fernández-Espínola, C., \& Almagro, B. J. (2019). Relation among motivation and emotional intelligence in Physical Education/ : A systematic review. Retos. Nuevas tendencias en Educación Física, Deporte y Recreación, 36, 584-589.

Fierro Suero, S., Almagro, B. J., \& Sáenz-López, P. (2019). Necesidades psicológicas , motivación e inteligencia emocional en Educación Física. Revista Electrónica Interuniversitaria de Formación del Profesorado, 22(2), 167-186. https: / /doi.org/http: / /dx.doi.org/ $10.6018 /$ reifop. 22.2.345241

Fierro-Suero, S., Almagro, J. A., \& Sáenz-López, P. (2020). Validation of the Achievement Emotions Questionnaire for Physical Education (AEQ-PE). International Journal of Environmental Research and Public Health, 17, 4560. https://doi.org/doi:10.3390/ ijerph17124560

Fierro-Suero, S., Almagro, J. A., \& Sáenz-López, P. (2021). Necesidades psicológicas básicas, emociones y satisfacción con la vida en docentes. Validación del AEQ-T en castellano. Artículo entregado para la publicación.

Frenzel, A. C., Goetz, T., Lüdtke, O., Pekrun, R., \& Sutton, R. E. (2009). Emotional Transmission in the Classroom: Exploring the Relationship Between Teacher and Student Enjoyment. Journal of Educational Psychology, 101(3), 705-716. https:// doi.org/10.1037/a0014695
Hombrados-Mendieta, I., \& Castro-Travé, M. (2013). Apoyo social, clima social y percepción de conflictos en un contexto educativo intercultural. Anales de Psicologia, 29(1), 108-122. https: / / doi.org/10.6018/ analesps.29.1.123311

Jiang, J., Vauras, M., Volet, S., \& Wang, Y. (2016). Teachers' emotions and emotion regulation strategies: Self- and students' perceptions. Teaching and Teacher Education, 54, 22-31. https://doi.org/ 10.1016/j.tate.2015.11.008

Jiménez Morales, M. I., \& López-Zafra, E. (2009). Inteligencia emocional y rendimiento escolar: Estado actual de la cuestión. Revista Latinoamericana de Psicologia, 41(1), 69-79.

Leisterer, S., \& Jekauc, D. (2019). Students' Emotional Experience in Physical Education-A Qualitative Study for New Theoretical Insights. Sports, 7(1), 10. https://doi.org/10.3390/sports7010010

López, L., \& Bisquerra, R. (2013). Validación y análisis de una escala breve para evaluar el clima de clase en educación secundaria. Psicopedagogía, 62-77.

Løvoll, H. S., Bentzen, M., \& Säfvenbom, R. (2020). Development of Positive Emotions in Physical Education: Person-Centred Approach for Understanding Motivational Stability and Change. Scandinavian Journal of Educational Research, 64(7), 9991014. https://doi.org/10.1080/ 00313831.2019 .1639818

Løvoll, H. S., Røysamb, E., \& Vittersø, J. (2017). Experiences matter: Positive emotions facilitate intrinsic motivation. Cogent Psychology, 4, 1340083. https: / / doi.org/10.1080/23311908.2017.1340083

Luo, W., Lim, S. Q. W., Choong, P. L., \& Liem, G. A. D. (2020). Perceived school goal structure, achievement goals and emotions in teaching of Singapore teachers. Asia Pacific Journal of Education, 40(3), 344-355. https://doi.org/10.1080/ 02188791.2019 .1705760

Martínez Múñoz, M. (2000). Programa de orientación del clima de clase. En M. Alvarez \& R. Bisquerra (Ed.), Manual de Orientación y tutoría. Barcelona: Praxis.

Mejía, A., \& Arturo, L. (2009). Relaciones sociales e interacción en el aula en secundaria. Revista Mexicana de Investigación Educativa, 14, 485-513.

Meyer, D. K., \&Turner, J. C. (2006). Re-conceptualizing emotion and motivation to learn in classroom contexts. Educational Psychology Review, 18(4), 377 390. https: / /doi.org/10.1007/s10648-006-9032-1 Moreno-Murcia, J. A., Huéscar, E., \& Ruiz, L. (2018). 
Perceptions of Controlling Teaching Behaviors and the Effects on the Motivation and Behavior of High School Physical Education Students. International Journal of Environmental Research and Public Health, 15, 2288. https: / / doi.org/10.3390/ijerph15102288

Mujica, F., \& Jiménez, A. (2021). Positive emotions of the students of Secondary Education in the practices of basketball in Physical Education. Retos. Nuevas tendencias en Educación Física, Deporte y Recreación, 39, 556 564. https: / / doi.org/10.47197/retos.v0i39.80112

Mujica, F., \& Orellana, N. (2021). Emociones del profesorado de educación física: revisión narrativa (20102020). Retos. Nuevas tendencias en Educación Física, Deporte y Recreación, 39, 910-914. https://doi.org/ 10.47197/retos.v0i39.80750

Mujica, F. (2021). Emociones negativas del alumnado de Secundaria en el aprendizaje de baloncesto en Educación Física. Retos. Nuevas tendencias en Educación Física, Deporte y Recreación, 41, 362-372. https:// doi.org/10.47197/retos.v0i41.84395

Musitu, G., \& Cava, M. (2003). El rol del apoyo social en el ajuste de los adolescentes. Intervención Psicosocial, 12(2), 179-192.

Pekrun, R., Lichtenfeld, S., Marsh, H. W., Murayama, K., \& Goetz, T. (2017). Achievement Emotions and Academic Performance: Longitudinal Models of Reciprocal Effects. Child Development, 88(5), 16531670. https: / / doi.org/10.1111/cdev. 12704

Pekrun, R. (2006). The control-value theory of achievement emotions: Assumptions, corollaries, and implications for educational research and practice. Educational Psychology Review, 18, 315-341. https: / / doi.org/10.1007/s10648-006-9029-9

Pekrun, R. (2014). Emotions and Learning. En Gonnet Imprimeur (Ed.), Unesco. https: / / doi.org/10.1080/ 00344087.2019.1669866

Pekrun, R, \& Stephens, E. J. (2010). Achievement Emotions: A Control-Value Approach. Social and Personality Psychology Compass, 4(4), 238-255. https: / /doi.org/10.1111/j.1751-9004.2010.00259.x

Pekrun, R., Goetz,T., Frenzel, A., Barchfeld, P., \& Perry, R. (2011). Measuring emotions in students' learning and performance: The Achievement Emotions Questionnaire (AEQ). Contemporary Educational Psychology, 36, 36-48. https://doi.org/10.1016/ j.cedpsych.2010.10.002

Pérez, A., Ramos, G., \& López, E. (2009). Diseño y análisis de una escala para la valoración de la variable clima social aula en alumnos de Educación Primaria y Secundaria. Revista de Educación, 350, 221 -
252.

Reeve, J. (2010). Motivación and Emoción. McGraw Hill: Mexico.

Reschly, A. L., Huebner, E. S., Appleton, J. J., \& Antaramian, S. (2008). Engagement as flourishing: The contribution of positive emotions and coping to adolescents' engagement at school and with learning. Journal of adolescence, 45(5), 419-431. https: // doi.org/10.1002/pits

Reyes, M. R., Brackett, M. A., Rivers, S. E., White, M., \& Salovey, P. (2012). Classroom Emotional Climate, Student Engagement, and Academic Achievement. Journal of Educational Psychology, 104(3), 700-712. https://doi.org/10.1037/ a0027268

Ryan, R. M., \& Deci, E. L. (2017). Self-Determination Theory. Basic Psychological Needs in Motivation, Development, andWelness. NewYork, NY, USA: Guilford Publications.

Simonton, K. L., \& Garn, A. (2019). Exploring Achievement Emotions in Physical Education: The Potential for the Control-Value Theory of Achievement Emotions. Quest, 71(4), 434-446. https: / /doi.org/10.1080/00336297.2018.1542321 Simonton, K. L., Garn, A. C., \& Solmon, M.A. (2017). Class-related emotions in secondary physical education: A control-value theory approach. Journal of Teaching in Physical Education, 36, 409-418. https:/ /doi.org/10.1123/jtpe.2016-0131

Siu, A. (2008). A Prevalence Study on Internalizing Problems Among Pri- mary School Children in Hong Kong. Journal of child and family studies, 17(6), 779790.

Vecina, M. (2006). Emociones positivas. Papeles del psicólogo, 27(1), 9-17.

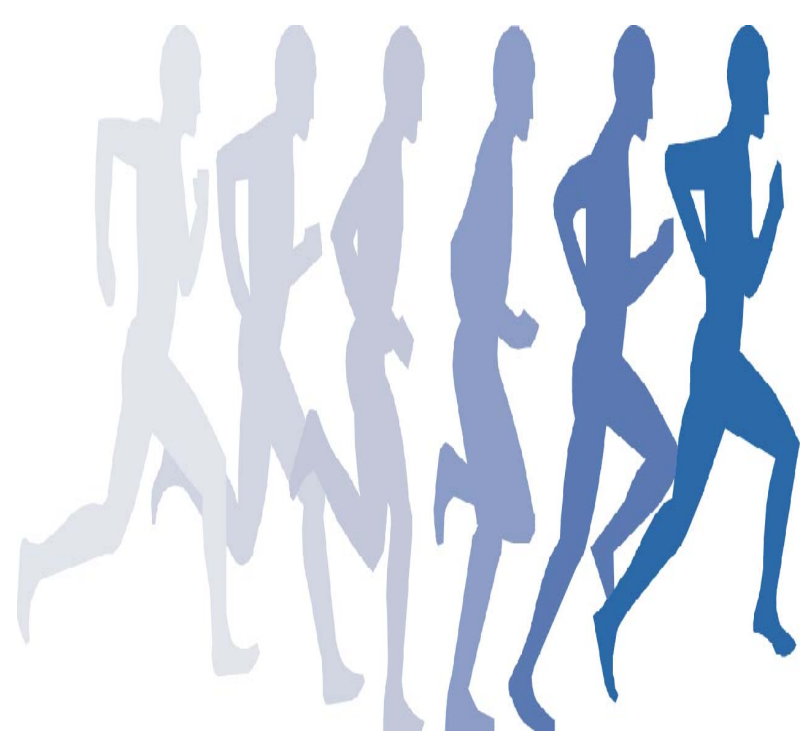

